\title{
Quantification of bed-load transport over dunes
}

\author{
Kenneth Lockwood ${ }^{1}$, Patrick Grover ${ }^{1}$, and Ana Maria Ferreira da Silva ${ }^{1}$ \\ ${ }^{1}$ Department of Civil Engineering, Queen's University, 58 University Av., Kingston ON, Canada
}

\begin{abstract}
There is disagreement in the literature as to whether a shear stress-based approach can be used to accurately predict sediment transport over dunes. This study aims to address this disagreement. To this end, use is made of an experiment involving the study of naturally formed, fully developed dunes produced in a laboratory flume. The bed shear stress is estimated through a combination of velocity, Reynolds stress measurements, and results of a CFD RANS rough wall model. The validity of using Bagnold's equation to predict the bed-load rate is subsequently analyzed. In contrast to what has been previously suggested by some authors, it is found from the present experiment that the bed-load rate correlates well with the bed shear stress, and that Bagnold's equation yields realistic values of the bed-load rate over the stoss side of the dune downstream of the reattachment point. This work also highlights the difficulties in reliably estimating the bed shear stress in complex flows. Such difficulties are overcome in this paper through a combination of flow velocity measurements and modeled results.
\end{abstract}

\section{Introduction}

Dunes are the most common type of bed forms occurring in sand rivers and have a significant impact on both hydraulic roughness and sediment transport. Owing to their practical and scientific significance, extensive research has been dedicated to dunes for many years. In spite of this, many aspects of dune dynamics, and especially the coupling of flow and sediment transport remains unclear. In this context, there appears to be disagreement on how to quantify local sediment transport rates over dunes. The matter acquires particular importance when calculating the downstream migration of dunes, or the morphological changes they experience under changing flow conditions. This paper, addressing the quantification of local sediment transport rate over dunes, is motivated by these considerations. The work is restricted to the case of bed-load transport.

As is well-known, existing equations for the quantification of bed-load rate (MeyerPeter, Bagnold, etc.) were developed for uniform flow conditions. Such equations invariably give bed-load rate as a function of bed shear stress. However, the flow over dunes is not only non-uniform, but also internally highly complex [1-13]. The flow suddenly expands downstream of the dune crest, it separates from the boundary, and reattaches to it at approximately 4 to 6 times the dune height. At the boundary of the separation zone there is a shear layer where vortices generate due to Kelvin Helmholtz instabilities. As the flow progresses downstream, an internal boundary layer develops with the flow accelerating over the stoss side of the dune. The question that arises is whether existing equations can represent bed-load rate in the accelerating region downstream of the reattachment point. For this to happen, the bed-load rate must be directly related to the local bed shear stress as the flow 
accelerates. On the basis of experimental results, it has been suggested by a number of authors that such an approach may not be appropriate $[5,6]$. On the other hand, in direct contradiction to such conclusions, several recent numerical models employing existing bed-load rate equations have produced results on dune morphological changes that compare favorably with experimental and field data [14-16]. Considering the aforementioned, the specific objective of the present work is to experimentally investigate the applicability of existing equations to predict bed-load rate over dunes outside of the recirculation zone.

\section{Present measurements}

For the present purposes an experiment was carried out in the $21 \mathrm{~m}$ long, $0.76 \mathrm{~m}$ wide, $0.5 \mathrm{~m}$ deep sediment transport flume at Queen's University. This experiment follows from previous work performed by Wiebe [17] in the same flume. In that study, flow was run over an initially flat mobile sand bed $\left(D_{50}=1.0 \mathrm{~mm}\right)$ for $268 \mathrm{~min}$ allowing dunes to form and grow until they were fully developed at the equilibrium state. The time to reach equilibrium was estimated as $238 \mathrm{~min}$. Longitudinal profiles of the dunes were acquired throughout the run using a Delft Hydraulics PV-09 profiler. The flow conditions were as given in Table 1. Here $Q$ is flow rate, $h$ is flow depth, $v_{*}$ is shear velocity $\left(=(g S h)^{0.5}\right.$, in which $g$ is acceleration due to gravity and $S$ is bed slope), $R e$ is flow Reynolds number ( $=u_{a v} h / v$, in which $u_{a v}$ is average flow velocity and $v$ is fluid kinematic viscosity), $F r$ is Froude number $\left(=u_{a v} /(g h)^{0.5}\right), R e_{*}$ is roughness Reynolds number $\left(=v_{*} k_{s} / v\right.$, in which $k_{s}$ is granular skin roughness), $\theta$ is Shields parameter (= $\left(\rho v_{*}^{2}\right) /\left(\gamma_{s} D\right)$, in which $\rho$ is fluid density, $\gamma_{s}$ is specific weight of grains in fluid and $D$ is a representative grain size, henceforth identified with $D_{50}$ ), and $\theta_{c r}$ is the value of $\theta$ at the critical stage of initiation of sediment transport. Examples of measured bed profiles are given in Fig. 1.

In the present study a train of 10 identical dunes were created using the average dimensions of the fully developed dunes from Wiebe [17]. That is, the dunes used in the present study are to be viewed as a periodic, idealized version of the equilibrium dunes produced by Wiebe [17]. The dunes were then immobilized using the sand-cement based method of Ebrahimi and da Silva [18] so as to enable the measurement of the flow field and turbulence characteristics over them. The immobilization method yields a coating layer with a granular roughness comparable to that of the original mobile bed. Flow velocity measurements were carried out using a Nortek Vectrino II Acoustic Doppler Velocimeter (ADV) with a sampling frequency of $20 \mathrm{~Hz}$ and a sampling duration of $3 \mathrm{~min}$. The sampling frequency used is in line with that used by other researchers in laboratory experiments over fixed dunes $[19,20]$. The sampling duration was deemed sufficient as time averaged velocity and Reynolds stress were found to converge after about $100 \mathrm{~s}$, well before the end of the sampling. The ADV collects a profile of the velocity field over a range between 4 and $7.5 \mathrm{~cm}$ below the probe with a sampling volume diameter of $6 \mathrm{~mm}$, and a height of individual sampling volumes that can be adjusted between 1 to $4 \mathrm{~mm}$. In this experiment the sampling volume was split into 11 cells with a $3 \mathrm{~mm}$ height. Measurements were taken at 12 verticals over the lee side of the $5^{\text {th }}$ dune and stoss side of the $6^{\text {th }}$ dune as shown in Fig. 2 . The data collected by the ADV was of high quality with a correlation index whose mean value was

Table 1. Summary of hydraulic conditions in the run by Wiebe [17] [ $B=0.76 \mathrm{~m} ; S=1 / 792]$.

\begin{tabular}{|c|c|c|c|c|c|c|}
\hline $\boldsymbol{Q}\left(\mathbf{m}^{\mathbf{3}} / \mathbf{s}\right)$ & $\boldsymbol{h}(\mathbf{m})$ & $\boldsymbol{v}_{*}(\mathbf{m} / \mathbf{s})$ & $\boldsymbol{R e}$ & $\boldsymbol{F r}$ & $\boldsymbol{R e}_{*}$ & $\boldsymbol{\theta} / \boldsymbol{\theta}_{\boldsymbol{c r}}$ \\
\hline 0.1365 & 0.320 & 0.063 & 179600 & 0.32 & 126 & 6.82 \\
\hline
\end{tabular}




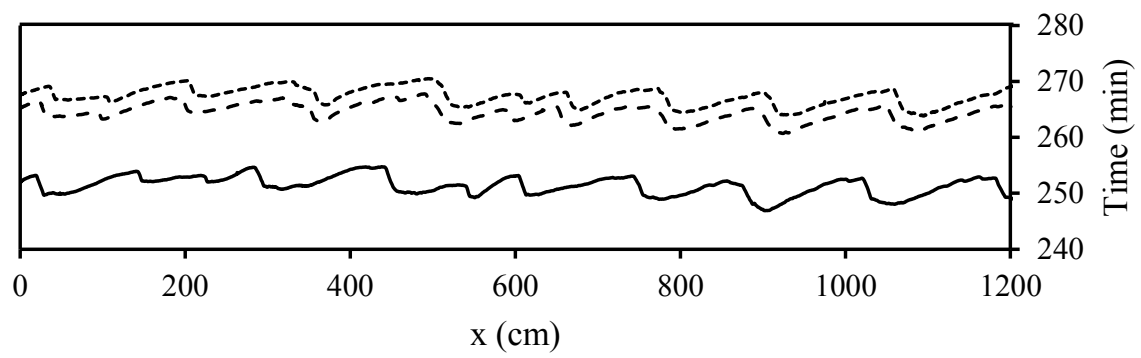

Fig. 1. Measured bed profiles (from Wiebe [17]); $x=0$ in this figure means the beginning of the measurement region (5.4 m downstream of the flume entrance). The solid, long-dashed and shortdashed profiles were measured at 252, 265 and $268 \mathrm{~min}$, respectively.

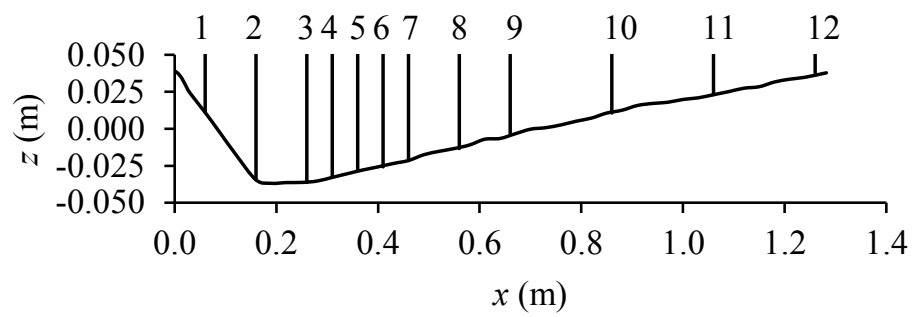

Fig. 2. Location of measurement verticals over the lee side of the fifth and stoss side of the sixth dune. The value $x=0$ in this figure corresponds to the crest of the fifth dune.

$84 \%$. Only $3.6 \%$ of the data was below the cut off threshold of $70 \%$ for reliable measurements [21]. Similarly, the mean signal to noise ratio was $46 \mathrm{db}$ with a minimum of $34 \mathrm{db}$, both well above the cut-off of $15 \mathrm{db}$ for reliable data [21]. Finally, it was found that the uncertainties in the mean velocity and Reynolds stress were $1 \%$ and $7 \%$, respectively, with a $95 \%$ confidence interval.

\section{Results}

\subsection{Flow field and turbulence characteristics}

The fields of measured time average longitudinal flow velocity and Reynolds stress $\tau_{u w}$ (= $\left.-\rho \overline{u^{\prime} w^{\prime}}\right)$ are shown in the contour plots in Fig. 3. Here $u^{\prime}$ and $w^{\prime}$ are the fluctuating components of longitudinal and vertical velocities, respectively. For more detail, the measured profiles of these quantities are shown in Fig. 4. The measured flow characteristics follow several well described patterns as discussed in the Introduction, with flow accelerating over the stoss side of the dune and reaching a maximum at the crest, flow separating over the lee side of the dune, negative flow velocities occurring within the recirculation zone created by flow separation, and high Reynolds stresses occurring at the boundary of the recirculation zone. To determine the upper boundary of the recirculation zone $(H)$, the near bed profiles of time averaged longitudinal flow velocity were integrated along $z$, with $H$ being the point at which $\int_{0}^{H} \bar{u} d z=0$. The reattachment point was found to occur at $x=0.40 \mathrm{~m}(=5.4 \Delta$, in which 

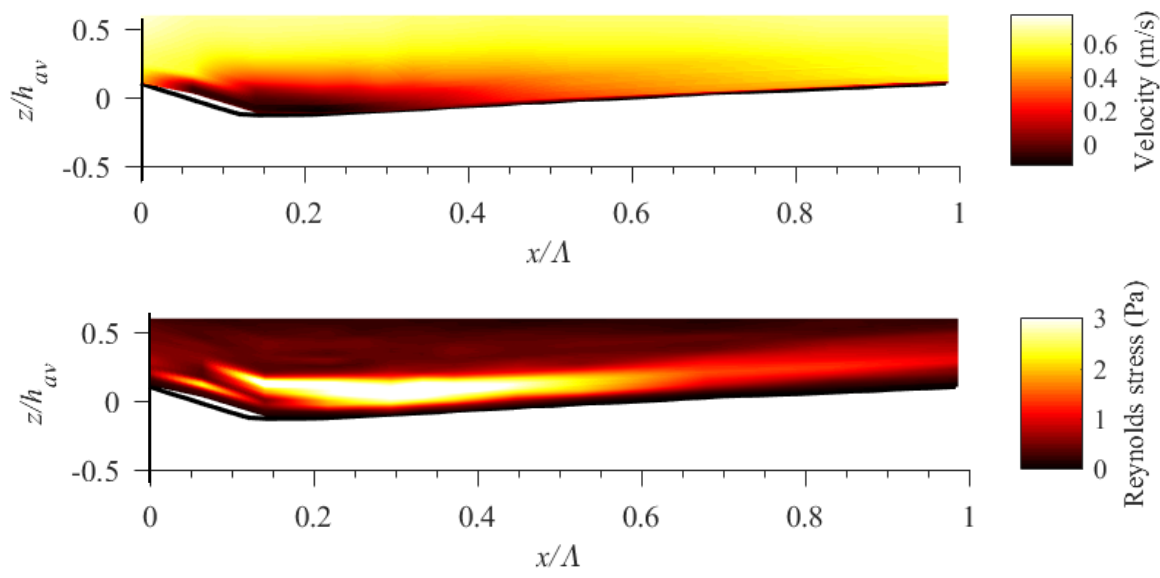

Fig. 3. Contour plots of longitudinal flow velocity (top) and Reynolds stress $\tau_{u w}$ (bottom).
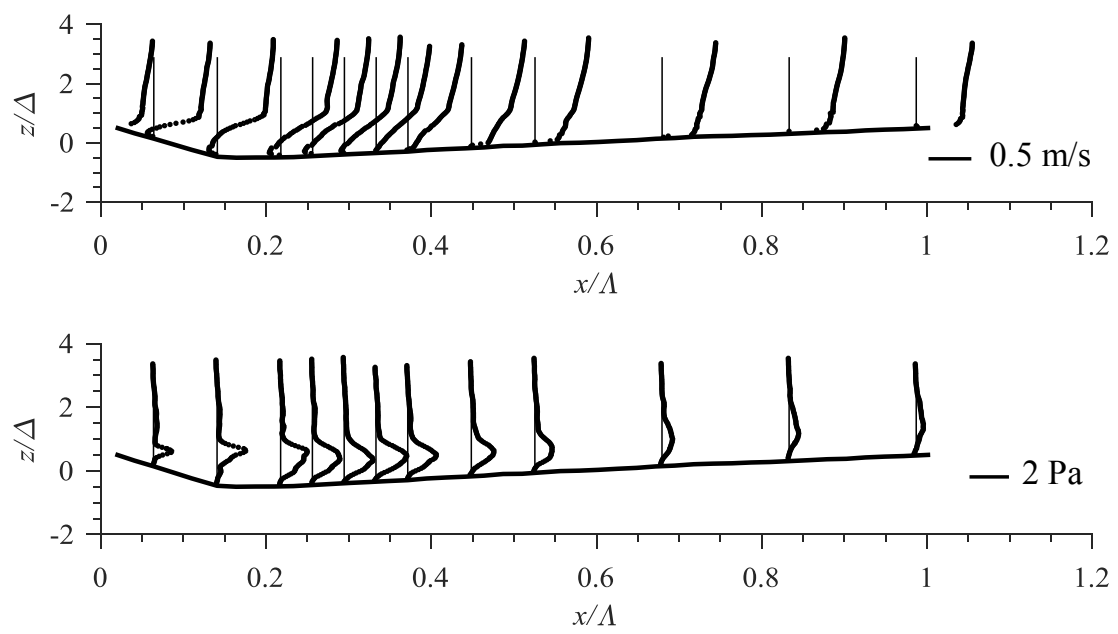

Fig. 4. Vertical profiles of longitudinal flow velocity (top) and Reynolds stress $\tau_{u w}$ (bottom).

$\Delta$ is dune height). This value falls within the range of four to six times the dune height found within literature values for reattachment length $[6,13,22,23]$.

\subsection{Bed shear stress and bed-load rate}

Because of limitations in measuring equipment, usually, as is the case in this work, the velocity measurements do not cover the region close to the bed. In the absence of such measurements, the bed shear stress is determined by extrapolating to the bed the profiles of pertinent quantities. If the velocity profile is logarithmic, there are well-known methods to do so, resting on the knowledge that shear stress is linearly distributed over the flow depth. However, in general the velocity profile over dunes deviates considerably from a logarithmic shape, and therefore such methods are no longer appropriate. In this work, the bed shear stress was determined on the basis of the following well known equation [24]: 


$$
\tau=\mu \frac{d u}{d z}-\rho \overline{u^{\prime} w^{\prime}}
$$

with $\tau_{0}=\tau_{z=0}$.

By considering the profiles of $-\rho \overline{u^{\prime} w^{\prime}}$, it was found that they are relatively well behaved near the bed, making it possible to accurately predict the value of $-\rho \overline{u^{\prime} w^{\prime}}$ at the bed. This is illustrated in Fig. 5 from which the values of $-\rho \overline{u^{\prime} w^{\prime}}$ at the bed were found to be 0.12 and $0.02 \mathrm{~Pa}$ for measurement verticals 5 and 9 (see Fig. 2). The same was not possible for $d u / d z$ as the lack of measurements within $3 \mathrm{~mm}$ of the bed made it impossible to resolve the large velocity gradients in the region. To overcome this difficulty, $d u / d z$ near the bed was determined from the flow field calculated by Grover and da Silva [25] for the conditions in the present experiment. These authors used a CFD RANS rough-wall model based on the $k$ $\omega$ SST turbulence closure. As demonstrated in Fig. 6 there is good agreement between the measured and calculated values of $d u / d z$ and hence the writers believe that this model yields a reasonable estimate of $d u / d z$ at the bed. A plot of the resulting values of $\tau_{0}$ along the pertinent dune is shown in Fig. 7.
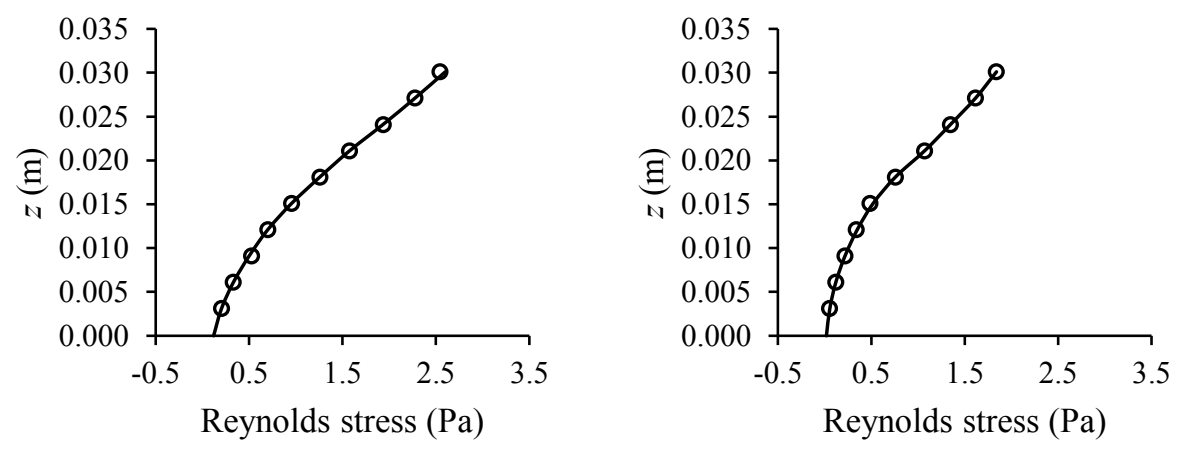

Fig. 5. Examples of extending profiles of Reynolds stress to the bed for measurement verticals 5 (left) and 9 (right). Open circles are measured values; solid lines are best fits to the data.
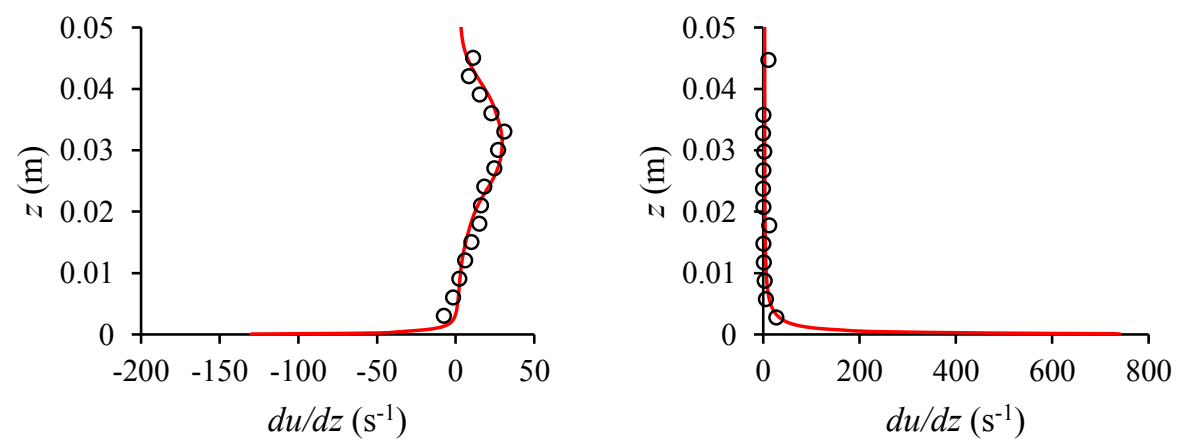

Fig. 6. Examples of extending profiles of $d u / d z$ to the bed for measurement verticals 1 (left) and 11 (right). Open circles are measured values; solid lines are the results of a RANS model. 


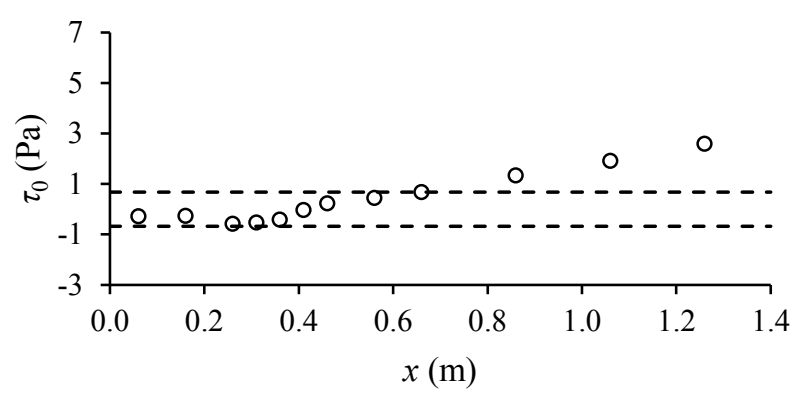

Fig. 7. Longitudinal distribution of $\tau_{0}$ along the dune of interest. The dotted lines mark the positive and negative values of critical shear stress. The reattachment point is at $x=0.4 \mathrm{~m}$.

Consider now Bagnold's bed-load rate equation [26]:

$$
q_{s b}=\frac{\beta u_{b}\left(\tau_{0}-\left(\tau_{0}\right)_{c r}\right)}{\gamma_{s}} .
$$

Here $q_{s b}$ is specific volumetric bed-load rate, $u_{b}$ is flow velocity at the bed, and $\beta$ is a coefficient which for the present flow conditions can be identified with 0.5. Following da Silva and Yalin [27], $u_{b}$ was calculated as $u_{b}=\left(u_{k s}+u_{\varepsilon}\right) / 2$ where $u_{k s}$ and $u_{\varepsilon}$ are the values of $u$ at $z=k_{s}$ and $z=\varepsilon$, respectively, with $\varepsilon$ being the height of the bed-load layer. This was calculated from the relation $\varepsilon / D=0.3 \Xi^{0.7}\left(\theta / \theta_{c r}-1\right)^{0.5}$ due to van Rijn [28], and in which $\Xi$ is the material number. The value of $\left(\tau_{0}\right)_{c r}$ was determined from Eq. (1.34) in da Silva and Yalin [27], which for $D=1 \mathrm{~mm}$ yields $\left(\tau_{0}\right)_{c r}=0.677 \mathrm{~Pa}$. The resulting values of $q_{s b}$ along the pertinent dune are plotted in Fig. 8.

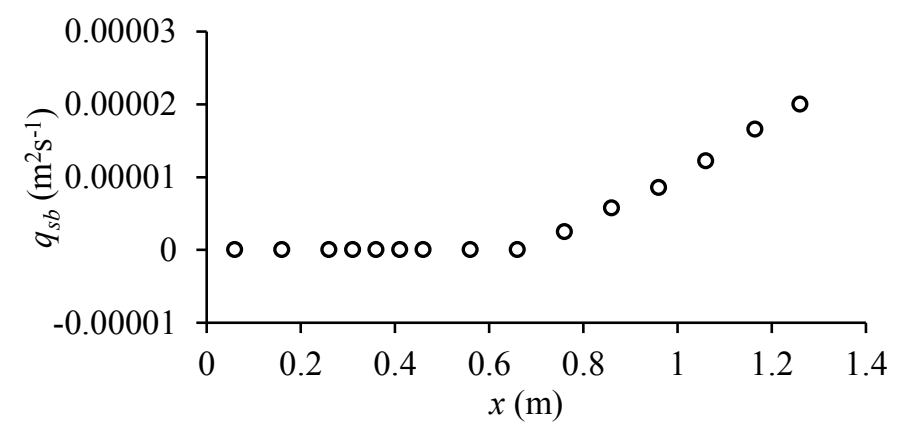

Fig. 8. Longitudinal distribution of $q_{s b}$ along the dune of interest.

\subsection{Coupling to Morphological Changes}

We proceed next to assess whether or not the bed-load rates calculated from Bagnold's equation are realistic. Since Wiebe [17] did not conduct bed-load rate measurements, it is not possible to base such assessment on a direct comparison between measured and calculated values of bed-load rate. Thus, in the following, we use an indirect approach, based on a comparison between measured and calculated dune morphological changes. Using the $q_{s b^{-}}$ 
values calculated in the previous section, changes in bed elevation were predicted using the sediment transport continuity equation, namely:

$$
(1-p) \frac{\partial z_{b}}{\partial t}=-\frac{\partial q_{s b}}{\partial x}
$$

where $z_{b}$ is bed elevation measured with regard to an arbitrary datum, $t$ is time and $p$ is porosity of the granular material forming the bed. In this work, for the $1.0 \mathrm{~mm}$ diameter sand, $p$ was identified with 0.3 . The predicted changes in bed elevation of the dune profile over a 13 min period are compared to the changes measured by Wiebe [17] over a similar period of time in Fig. 9. As can be seen from this figure, the predicted values match the measured results remarkably well outside of the recirculation zone. This suggests that it is indeed reasonable to use a shear-stress based approach to predict sediment transport over dunes. This is in agreement with the approach adopted by Tjerry and Fredsøe [14], Paarlberg et al. [15] and Niemann [16] in their numerical calculations of bed morphological changes.

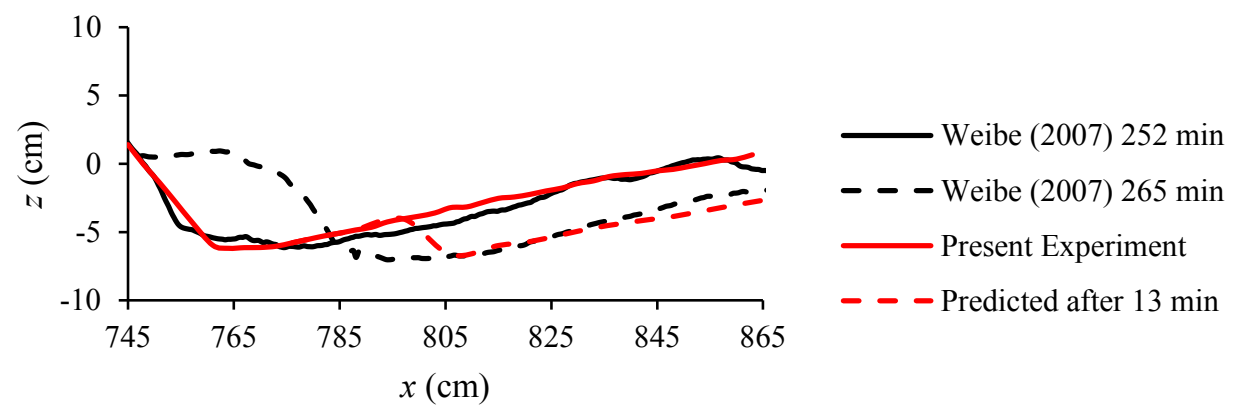

Fig. 9. Dune profiles measured by Wiebe [17] at two different times plotted together with dune profile in the present measurements and predicted changes to this profile.

\section{Conclusions}

The main finding of the present work is that downstream of the reattachment point, where the bed shear stress continuously increases over the stoss side of a dune, the bed-load rate correlates well with bed shear stress. The work further suggests that the existing bed-load rate equations yield realistic values of bed-load rate in the region between the reattachment point and the dune crest.

\section{Acknowledgements}

This research was supported by the Natural Sciences and Engineering Research Council of Canada (NSERC) through a Discovery Grant provided to the third author and an Alexander Graham Bell Canada Graduate Scholarship provided to the first author.

\section{References}

1. S.R. McLean, J.D. Smith, J. Geophys. Res., 84, 7791- 7808 (1979)

2. S.R. McLean, J.D. Smith, J. Hydr. Engrg., 112, 300-317 (1986)

3. J.M. Nelson, J.D. Smith, J. Geophys. Res., 94, 8146- 8162 (1989)

4. S.R. McLean, Earth Sciences Review, 29, 131- 144 (1990)

5. J.M. Nelson, S.R. McLean, S.R. Wolfe, Water Resour. Res., 29, 12, 3935- 3953 (1993) 
6. S.R. McLean, J.M. Nelson, S.R. Wolfe, J. Geophys. Res., 99, C6, 12729-12747 (1994)

7. S.R. McLean, S.R. Wolfe, J.M. Nelson, J. Hydr. Engrg., 125, 7, 725- 736. (1999a)

8. S.R. McLean, S.R. Wolfe, J.M. Nelson, J. Geophys. Res., 104, 15,743-15,753 (1999b)

9. S.J. Bennett, J.L. Best, Sedimentology, 42, 491- 513 (1995)

10. T.B. Maddux, J.M. Nelson, S.R. McLean, J. Geophys. Res., 108, F1, 6009, doi:10.1029/2003JF000017 (2003)

11. J.S. Bridge, Rivers and Floodplains: Forms, Processes and Sedimentary Record, Blackwell, Malden, Mass. (2003)

12. M.G. Kleinhans, Earth Sciences Reviews, 65, 75-102 (2004)

13. J. Best, J. Geophys. Res., 110, F04S02, doi:10.1029/2004JF000218 (2005)

14. S. Tjerry, J. Fredsøe, J. Geophys. Res., 110, F4, p. F04013 (2005)

15. A.J. Paarlberg, C.M. Dohmen-Janssen, S.J.M.H. Hulscher, P. Termes, J. Geophys. Res., 114, F1, p. F01014 (2009)

16. S.L. Niemann, J. Fredsøe, N.G. Jacobsen, J. Hydr. Engrg., 137, 1, 5-14 (2011)

17. J. Wiebe, Time and patterns of development of dunes subjected to sudden changes in flow depth. M.Sc Thesis, Queen's University, Kingston CA (2007)

18. M. Ebrahimi, A.M.F. da Silva, J. Hydr. Res., 51, 3, 306-316 (2013)

19. J.G. Venditti, S.J. Bennett, J. Geophys. Res., 105, 9, 22,035-22,047 (2000)

20. J.G. Venditti, B.O. Bauer, Earth Surf. Proc. and Landforms, 30, 289-304 (2005)

21. P.J. Rusello, A. Lohrmann, E. Siegel, T. Maddux, Proc. 7th Int. Conf. on Hydroscience and Engineering, Philadelphia, USA (2006)

22. R. Balachandar, V.C. Patel, Canadian J. Civil Engrg., 34, 12, 1587-1599 (2007)

23. R. Balachandar, V.C. Patel, Canadian J. Civil Engrg., 35, 5, 511-520 (2008)

24. S.B. Pope SB, Turbulent flows, Cambridge University Press, UK (2000)

25. P. Grover, A.M.F. da Silva, Proc. River Flow 2016, $8^{\text {th }}$ Int. Conf. on Fluvial Hydraulics, CRC Press, Taylor \& Francis Group, London, ISBN 978-1-138-02913-2, 1092-1097 (2016)

26. R.A. Bagnold, Philos. Trans. Royal Soc. of London, A, Mathematical and Physical Sciences, 249, 964, 235-262 (1956)

27. A.M.F. da Silva, M.S. Yalin, Fluvial Processes: 2 nd edition, IAHR Monograph Series, CRC Press/Balkema, Taylor \& Francis Group, The Netherlands (2017)

28. L.C. van Rijn, J. Hydr. Engrg., 110, 11, 1613-1641 (1984) 